\title{
Temperature Sensitivity of the Preoptic and Anterior Hypothalamic Neurons in Organ Gulture
}

\author{
Yayoi Hori and Teruo Nakayama \\ Department of Physiology, Osaka University Medical School, \\ Osaka 530
}

\begin{abstract}
Hori, Y. and Nakayama, T. Temperature Sensitivity of the Preoptic and Anterior Hypothalamic Neurons in Organ Culture. Tohoku J. exp. Med., 1982, 136 (1), 79-87 _ Single unit activities were recorded from the preoptic and anterior hypothalamic $(\mathrm{POAH})$ explants obtained from newborn mice brain to study neural temperature sensitivities in the period of 10-34 days culture. The firing rate of recorded units was less than 13 impulses per sec at $35^{\circ} \mathrm{C}$. Fifteen warm sensitive, 2 cold sensitive and 37 thermally insensitive units were obtained. The temperature sensitivities of the cultured POAH neurons were similar to those of in vivo neurons, i.e. the discharge frequencies changed smoothly in proportion to $1-5^{\circ} \mathrm{C}$ changes in local temperature. The thermal responses of warm sensitive units increased after 18 days and decreased over 30 days in vitro. Two warm sensitive neurons were identified and observed under the optical microscope. The cold sensitive units were unable to be recorded until about 20 days in vitro. It became evident that the neural temperature sensitivities of $\mathrm{POAH}$ neurons were maintained in an absence of the extrahypothalamic afferents. - . temperature-sensitive neurons; hypothalamus; organ culture; temperature regulation
\end{abstract}

Thermally sensitive units which respond to changes in local brain temperature were first detected in the preoptic and anterior hypothalamic area (POAH) by Nakayama et al. in 1961. Warm sensitive units increased firing rates under local heating, while cold sensitive units increased firing rates under local cooling. POAH thermally sensitive units have been found in many mammals including cat (Nakayama et al. 1963; Wit and Wang 1968), dog (Hardy et al. 1964), rabbit (Nakayama and Hardy 1969), rat (Murakami 1973), ground squirrel and guinea pig (Boulant and Bignall 1973). In addition, behavioral thermo-regulation can be evoked in reptile (Myhre and Hammel 1969) and fish (Hammel et al. 1969) by heating and cooling the POAH. Even these ectotherms seemed to have thermally sensitive neurons.

The thermally sensitive neurons are influenced by extrahypothalamic thermal signals (Guieu and Hardy 1971) from the brain stem (Nakayama and Hardy 1969), medulla oblongata (Inoue and Murakami 1976), spinal cord (Guieu and Hardy 1970; Boulant and Hardy 1974) and skin (Boulant and Bignall 1973; Nakayama et al. 1979). Furthermore, non-thermal signals from the other parts of the nervous system might modulate to some extent the activity of thermally sensitive

Received for publication, February 24, 1981. 
units. It is of interest what characteristics can be observed in thermally sensitive units of $\mathrm{POAH}$ when they are deprived of all these extrahypothalamic afferents. An attempt was carried out to record the thermal characteristics of neurons from dissociated hypothalamic culture by Mason et al. (1978), wherein discharges appeared transiently during temperature changes. These findings were not in agreement with the behavior of discharges observed in vivo by Nakayama et al. (1963).

In our previous short paper (Nakayama et al. 1978), we presented the thermosensitivity of $\mathrm{POAH}$ neurons in the absence of extrahypothalamic afferents, using an organ culture method which kept cell membrane more intact than dissociated culture methods. The present study was made to observe the morphological and functional characteristics of $\mathrm{POAH}$ temperature sensitive neurons and to clarify the development of thermo-sensitivities in organ culture.

\section{Materials and Methods}

Organ culture of the hypothalamus. Hypothalamic explants were prepared from newborn ICR mice of both sexes by free-hand dissection under sterile conditions. The method of organ culture of the hypothalamus was described in our previous report (Nakayama et al. 1978). The procedure to get the subdivisional hypothalamic tissue was described by Masurovsky et al. (1971). This subdivisional hypothalamic tissue was dissected into 4 coronal sections about 300-500 $\mu \mathrm{m}$ thickness from rostrum to cauda and the residual hypothalamic tissue was discarded. Each coronal section was further symmetrically divided into 2 explants $(0.6 \mathrm{~mm}$ wide $\times 0.8 \mathrm{~mm}$ long $)$ through the third ventricle. Eight hypothalamic explants were obtained from one mouse. These 4 coronal sections consisted of preoptic area including diagonal bundle (Section 1), rostral part of anterior hypothalamus (Section 2), caudal part of anterior hypothalamus (Section 3) and ventromedial hypothalamus including infundibula (Section 4). Two explants placed on a collagen-coated cover slip (Bornstein 1958) with one drop of the medium were maintained in a Maximow double coverslip assembly at $36.5^{\circ} \mathrm{C}$. The medium was changed every 2 or 3 days and regular observation was done every day under a light microscope with long distance oil immersion lens (Zeiss $\times 40$ ). The medium was composed of 9 parts Gey's balanced salt solution (BSS), 9 parts Eagle's basal medium, 9 parts horse serum (PerFreez), 9 parts $50 \%$ of 9 days chick embryo extract and 2 parts of $10 \%$ glucose (final concentration $600 \mathrm{mg} / 100 \mathrm{ml}$ ) (Yonezawa 1961). The medium was saturated with $95 \% \mathrm{O}_{2}$ and $5 \% \mathrm{CO}_{2}$ and $\mathrm{pH}$ was adjusted to 7.4 .

Recording of neural activity. Glass-microelectrodes filled with $3 \mathrm{M}-\mathrm{NaCl}$, having resistance of 5-25 M $\Omega$ were used. Experimental arrangement is shown in Fig. 1. A glass microelectrode was brought gradually close to a neuron with a three-dimensional oil manipulator under the observation with an inverted microscope. Spontaneous neural activities were fed into a high input-impedance preamplifier and observed on an oscilloscope with sound monitoring, while the alternating current was eliminated by a rejection filter. The number of unit discharge per sec counted by a pulse counter and the medium temperature were recorded simultaneously with a two-channel recorder.

Control of medium temperature. The medium temperature was controlled by 4 Peltier thermoelectric modules which constituted a cooling and warming divice surrounding the chamber (Fig. 1). The chamber was placed on the stage of the inverted microscope. BSS of $2.5 \mathrm{ml}$ was used as medium. The medium temperature was confined below $37^{\circ} \mathrm{C}$, because cultured neurons and collagens easily reached a critical condition when the temperature rose above $37^{\circ} \mathrm{C}$. The rate of medium temperature change was less than $3^{\circ} \mathrm{C}$ per min. The temperature was measured with a copper-constantan thermocouple.

Estimation of explant temperature. As the thermocouple was located $10 \mathrm{~mm}$ apart from 


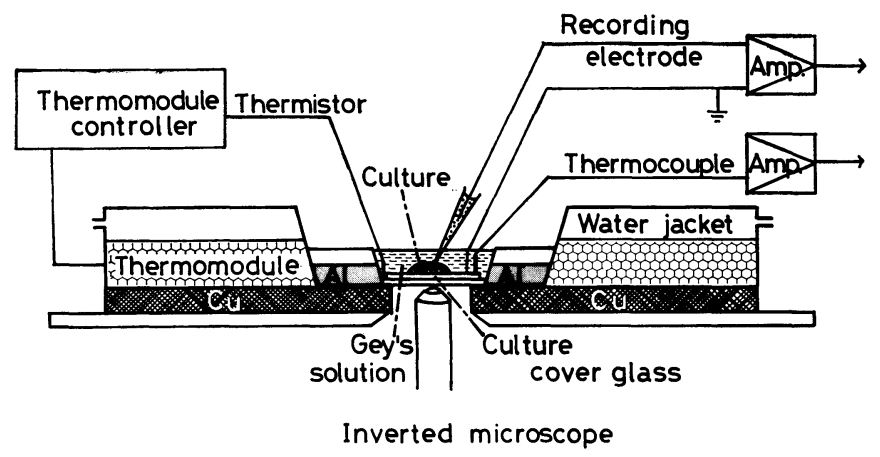

Fig. 1. Experimental arrangement for recording neuron activity from an explant during thermal stimulation. Four thermomodules (Peltier module) were controlled by a feed back system, fed with the medium temperature monitored with a thermistor.

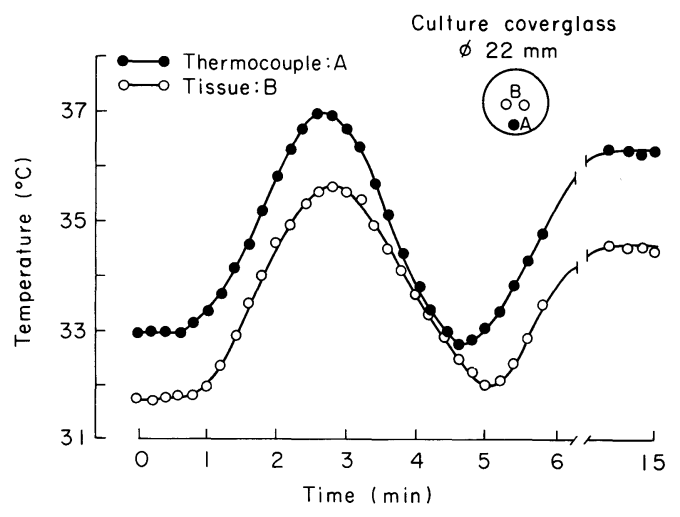

Fig. 2. Estimation of a true tissue temperature. Temperatures at A and B were simultaneously measured by thermocouples. Each explant (B) was located $1 \mathrm{~mm}$ apart from the center of the culture cover glass.

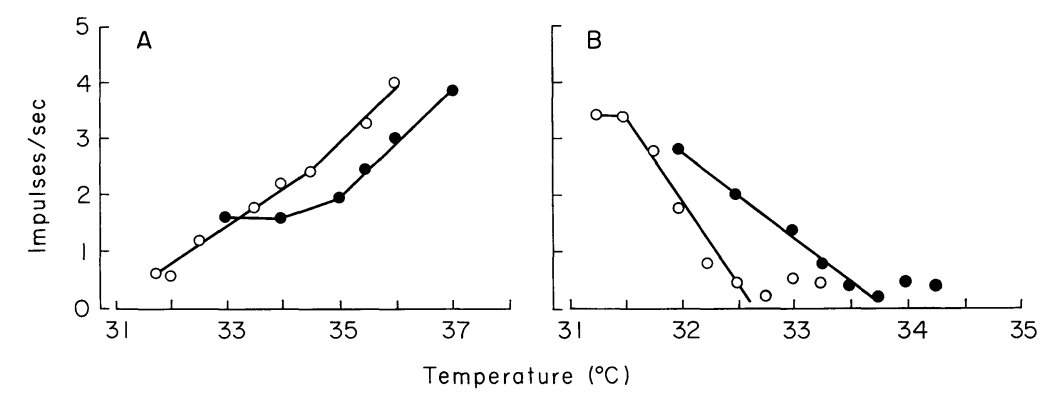

Fig. 3. Firing rate of a warm sensitive neuron (A) and a cold sensitive neuron (B) plotted against the thermocouple temperature and the estimated neuron temperature in the hypothalamic explant.

$\bullet-\bullet$, thermocouple temperature; $\bigcirc-0$, estimated temperature. 
the explants, true temperature of the explants always showed to be lower by about $1.5^{\circ} \mathrm{C}$ than the thermocouple temperature. In addition, there was about 15 sec delay during warming and cooling cycle between the temperature of thermocouple and explants as shown in Fig. 2. The impulse frequencies plotted against the measured temperature and the estimated explant temperature are shown in Fig. 3. The slope of the corrected curve turned out to be more linear (Fig. $3 \mathrm{~A}$ ) or more sharp (Fig. $3 \mathrm{~B}$ ) than that of the uncorrected curve. In the following figures, corrected temperatures were used.

\section{Results}

General morphological features of hypothalamic organ culture. Four hundred explants from 64 newborn mice were prepared for the hypothalamic cultures. Shapes of neural cells were not clearly observed with optical microscope in the early stages of culture. Necroses due to the tissue cutting became apparent in 2 days in vitro in the central zones of the explants. Subsequently, the explants recovered from partial necroses by the 7th to the 10th day of cultivation and then shapes of neural cells became gradually visible. Myelination took place in Section 1. This area was almost free of ependymal cells of the third ventricle wall. A round nucleus occupied almost entire area of the neural soma. Cells were round or slightly ovoid in form, about $10-15 \mu \mathrm{m}$ in diameter. The granules were rich in the cytoplasm and distributed surrounding the nucleus. Sections 2, 3 and 4 rarely showed myelination. Even when myelination of neural fibers occurred, these were formed unclearly and briefly on the dorsal or lateral surface of explants. The wall of the third ventricle with ependymal cell lying on one side of the square explant was clearly visible in sections 2,3 and 4 , and was used to identify the localization of explants. Cell bodies in which granules were diffusely distributed were ovoid in form and about $10-17 \mu \mathrm{m}$ in diameter (Fig. 4). In the arcuate nucleus, cells were slightly smaller than in the anterior hypothalamic area and were round or ovoid in form. The neural soma was rather large and had a round nucleus located in the center of the cell.

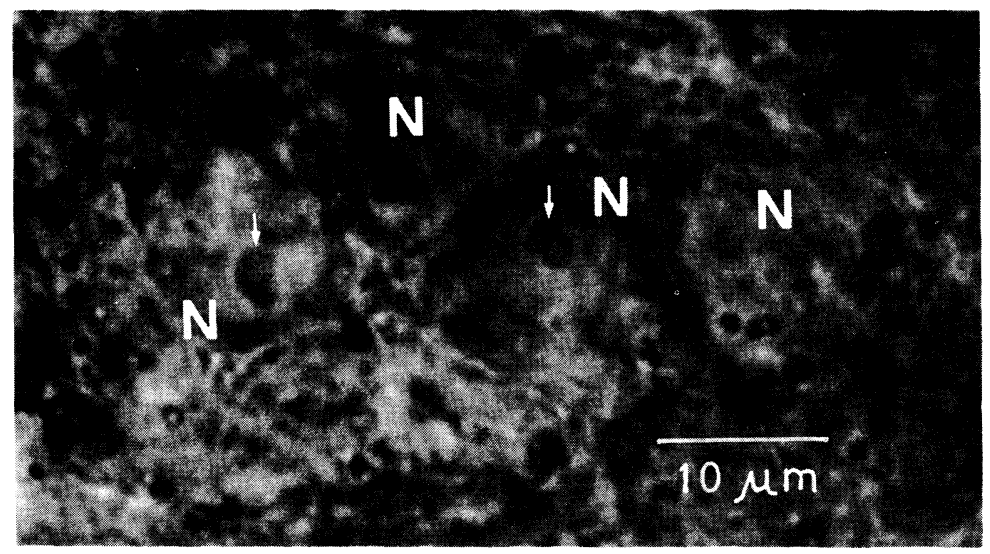

Fig. 4. Photomicrograph of neurons $(\mathrm{N})$ in the unstained mouse hypothalamic explant of Section 3 (29 days in vitro). Arrows show nucleoli in round nuclei. 
Morphological observations and location of thermally sensitive and insensitive neurons. The thermally sensitive units in cultured tissue in Sections 2, 3 and 4 were located more than $150 \mu \mathrm{m}$ lateral from the wall of the third ventricle and did not exist in the arcuate nucleus. Two warm sensitive neurons could be identified. They were ovoid in shape, $7.5 \times 17 \mu \mathrm{m}$ and $10 \times 15 \mu \mathrm{m}$ in size, respectively. Granules were diffusely distributed in them. The morphological differences between thermally sensitive and insensitive neurons were not detected through the light microscopic observation.

Classification of recorded unit activities. POAH neurons cultured 10 to 34 days were used for recording unit activities. For this purpose, 76 well conditioned explants which displayed minimum tissue damage and in which neural shapes could be clearly observed under the light microscope were selected. Of the recorded 54 units, 15 units were warm sensitive, 2 units were cold sensitive, while the remaining 37 units were thermally insensitive. Impulse frequency higher than 25 per sec was not observed within the corrected temperature range of $31-37^{\circ} \mathrm{C}$. In the frequency histogram at corrected temperature of $35^{\circ} \mathrm{C}, 91$ per cent of the thermally insensitive units fired less than 5 impulses per sec but the firing rate of warm sensitive units showed relatively uniform distribution in the range of 1-12 impulses per sec (Fig. 5). The firing rate of two cold sensitive units was less than 2 impulses per sec at corrected temperature of $35^{\circ} \mathrm{C}$.

Fig. 5. The histogram of discharge frequency of $\mathrm{POAH}$ neurons at corrected temperature of $35^{\circ} \mathrm{C}$.
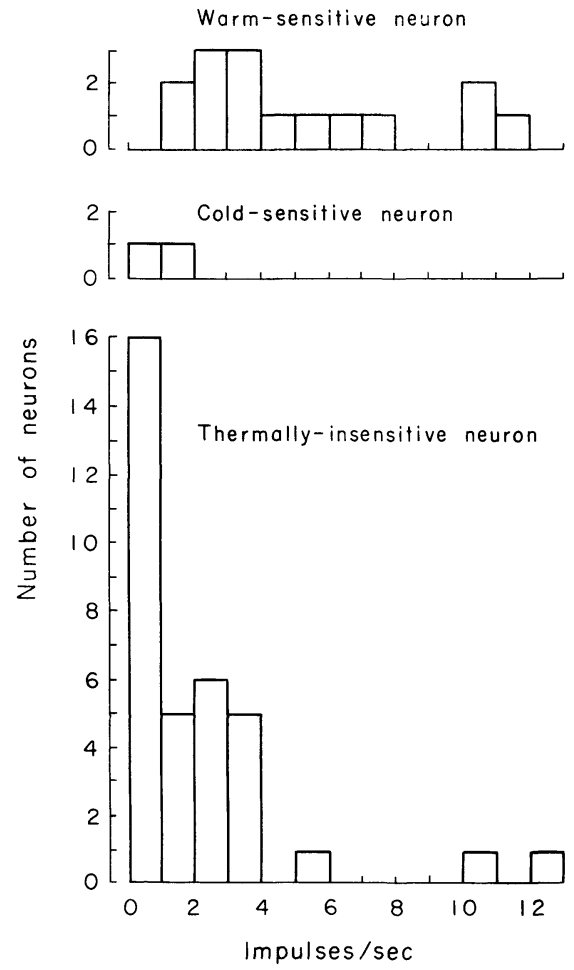
The firing patterns of the thermally sensitive units in vitro. Two types of thermal response were observed. One was of a warm sensitive neuron, the firing rate of which increased during warming and decreased during cooling (Fig. 6). The other was of a cold sensitive neuron which increased firing rate during cooling and decreased during warming (Fig. 7). These neural activities in cultured tissue were in agreement with the behavior of discharges observed in vivo, in a sense that the discharge frequencies changed smoothly in proportion to $1-5^{\circ} \mathrm{C}$ changes in local temperature and showed no dynamic response.

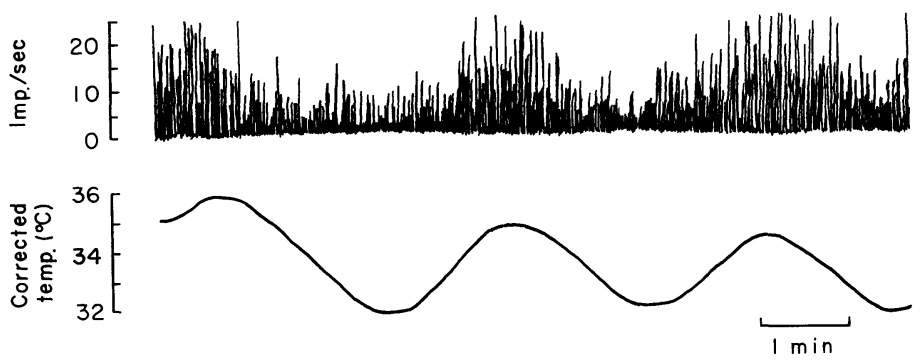

Fig. 6. Response of a warm sensitive neuron recorded from a hypothalamic explant. The explant was cultured for 22 days in vitro.

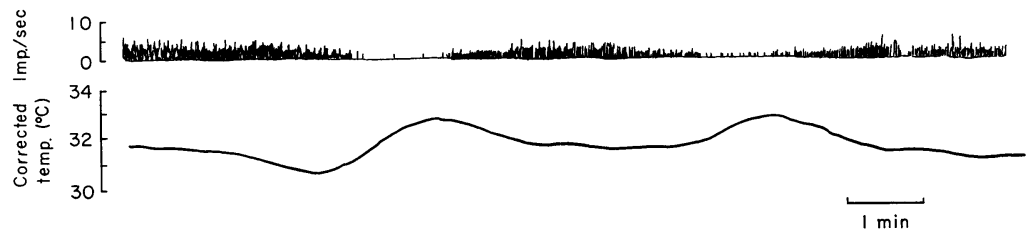

Fig. 7. Response of a cold sensitive neuron recorded from a hypothalamic explant. The explant was cultured for 26 days in vitro.

The temperature response of units with relation to days in culture. The steady state responses of cultured neurons to corrected tissue temperature are shown in Fig. 8. The discharge frequencies of 7 warm sensitive neurons studied on 18th to 29th day were more than 5 impulses per sec at corrected temperature of $35^{\circ} \mathrm{C}$ and their $Q_{10}$ ranged from 16 to 24 except for one neuron. In 4 warm sensitive neurons studied on 12th to 17th day and other 4 neurons on 30th to 34th day, the impulse frequencies were less than 5 per sec at corrected temperature of $35^{\circ} \mathrm{C}$ and their $Q_{10}$ were 2 to 8 and 2 to 12 , respectively. Only 2 cold sensitive units were obtained from the 2 explants cultured for 23 and 26 days as illustrated in Fig. 8 B. Increase of firing rates occurred when the corrected temperature was lowered below about $33^{\circ} \mathrm{C}$ in both units. On the other hand, thermally insensitive units had no noticeable correlation between firing rates and cultivated days (Fig. 8C). 


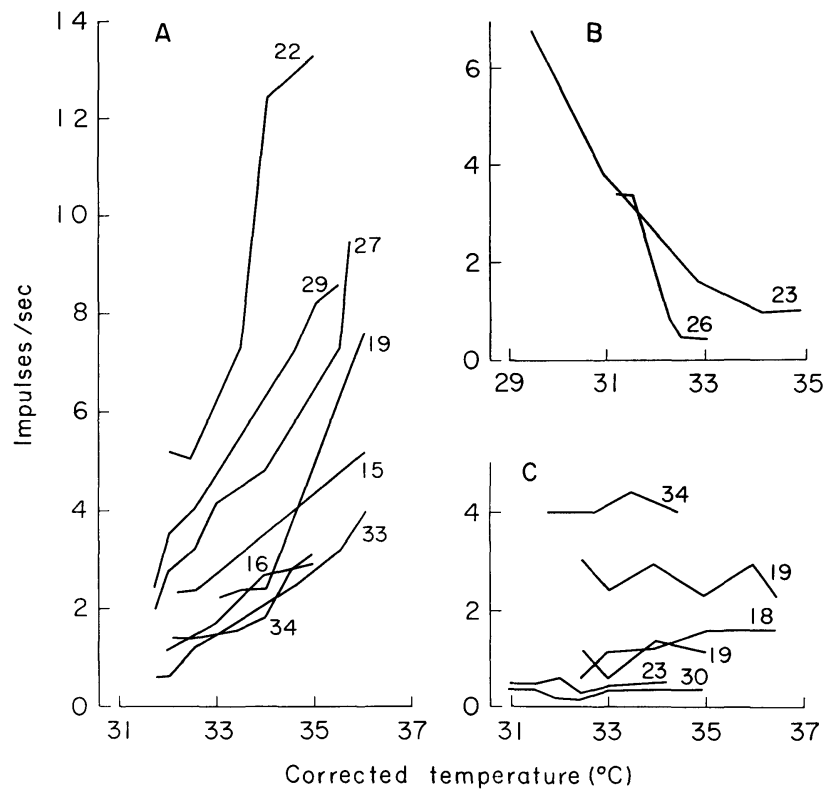

Fig. 8. The firing rates of warm sensitive (A), cold sensitive (B), and thermally insensitive (C) neurons in POAH explants plotted against the corrected temperature. Number on curves indicates the days in culture.

\section{Discussion}

In the present study, we used POAH organ cultures to identify thermally sensitive neurons. Two types of thermal response were observed, i.e. warm and cold sensitive units which increased discharge frequencies in response to warming and cooling, respectively. The $\mathrm{POAH}$ neurons still retained normal temperature sensitivity in organ culture from which extrahypothalamic afferents were eliminated (Figs. 6 and 7). This evidence indicates clearly that the thermally sensitive neurons exist in the POAH.

Using 76 explants, 15 warm sensitive units, 2 cold sensitive units and 37 thermally insensitive units were recorded. This result that the warm sensitive units are much more numerous than the cold sensitive units in POAH confirms the data of our previous short paper with organ culture (Nakayama et al. 1978) and the data of in vivo study (Hardy et al. 1964).

The firing rates in the POAH explants were less than 25 impulses per sec and significantly lower than those in vivo (Nakayama et al. 1979). For example, POAH unit discharges as many as 30 impulses per sec were demonstrated in cat (Nakayama et al. 1963). The low firing rate in vitro was reported by Hori et al. (1980) with the $\mathrm{POAH}$ slices of rat, in which the units fired at frequencies less than 15 impulses per sec at $38^{\circ} \mathrm{C}$. Their observation is consistent with our results in that the units fired less than 13 impulses per sec at $35^{\circ} \mathrm{C}$. In addition, the pattern of frequency distribution in the firing rate (Fig. 5) is comparable to Geller's (1975) 
observation on cultured hypothalamic neurons in rats. These results of the lower firing rate on isolated hypothalamic neurons lead us to the idea that such differences in discharge frequencies may be due to lack of extrahypothalamic afferents. Further support to the idea was given by the fact that thermally sensitive neurons in $\mathrm{POAH}$ were generally activated by extrahypothalamic afferent signals (Wit and Wang 1968; Nakayama and Hardy 1969; Guieu and Hardy 1970; Boulant and Hardy 1974; Inoue and Murakami 1976; Nakayama et al. 1979), i.e. Boulant and Hardy (1974) proposed that the only those warm sensitive units having high firing rates of greater than 10 impulses per sec received peripheral thermal input, while the cold sensitive units received those inputs in spite of discharge frequencies. At present, however, other possibilities such as species difference are not excluded.

One of the purposes of this study is to clarify the development of neural temperature sensitivities in culture. Our results showed that the temperature sensitivities of POAH neurons were closely related to days in culture (Fig. 8). After 18 days in culture, the warm sensitive units exhibited increased firing rates and $Q_{10}$ values in contrast to those of $12-17$ days in vitro. Furthermore, the cold sensitive unit was recorded first on the 23rd day of culture. Hori and Shinohara (1979) demonstrated in newborn rats that the firing rate of warm sensitive units increased during the first 20 days in postnatal rats and the warm sensitive units were detected earlier in the neonatal stage than the cold sensitive one. According to the morphological studies of hypothalamic neurons both in vivo (Reier et al. 1977) and in organ culture (Masurovsky et al. 1971), increase in number of synapses and advances in degree of maturity are evident in hypothalamic neurons for about one month. The development of the warm and cold sensitivities might be maturation. Cold sensitive neurons might require much longer time for neural maturation and synaptogenesis. However, the later appearance of cold sensitive units might be due to merely fewer recording chance both in vivo and in vitro, for the cold sensitive units are less in population than the warm sensitive one (Hardy et al. 1964; Nakayama et al. 1978; Hori et al. 1980). Observation after 30 days in vitro showed reduced firing rates and decreased $Q_{10}$ values of warm sensitive units (Fig. 8A). The reduced neural activity after 30 days in vitro of warm sensitive units cannot be explained merely as a result of general functional reduction in organ culture, because thermally insensitive units retained the same firing rate through the cultivated period. This fact suggests the possibility that neural network developed during culture may reduce the firing rates of warm sensitive units in vitro.

Only 2 neurons out of 15 warm sensitive units were identified and their sizes were measured under the microscope. The POAH explants will be useful for further studies on physiological and morphological characteristics of thermally sensitive neurons.

\section{References}

1) Bornstein, M.B. (1958) Reconstituted rat-tail collagen used as substrate for tissue cultures on coverslips. Lab. Invest., 7, 134-137. 
2) Boulant, J.A. \& Bignall, K.E. (1973) Hypothalamic neuronal responses to peripheral and deep-body temperatures. Amer. J. Physiol., 225, 1371-1374.

3) Boulant, J.A. \& Hardy, J.D. (1974) The effect of spinal and skin temperatures on the firing rate and thermosensitivity of preoptic neurones. J. Physiol., 240, 639-660.

4) Geller, H.M. (1975) Phasic discharge of neurons in long-term cultures of tuberal hypothalamus. Brain Res., 93, 511-515.

5) Guieu, J.D. \& Hardy, J.D. (1970) Effects of heating and cooling of the spinal cord on preoptic unit activity. J. appl. Physiol., 29, 675-683.

6) Guieu, J.D. \& Hardy, J.D. (1971) Integrative activity of preoptic units. I: Response to local and peripheral temperature changes. J. Physiol. (Paris), 63, 253-256.

7) Hammel, H.T., Strømme, S.B. \& Myhre, K. (1969) Forebrain temperature activates behavioral thermoregulatory response in Arctic Sculpins. Nature, 165, 83-85.

8) Hardy, J.D., Hellon, R.F. \& Sutherland, K. (1964) Temperature-sensitive neurones in the dog's hypothalamus. J. Physiol., 175, 242-253.

9) Hori, T. \& Shinohara, K. (1979) Hypothalamic thermo-responsive neurones in the new-born rat. J. Physiol., 294, 541-560.

10) Hori, T., Nakashima, T., Hori, N. \& Kiyohara, T. (1980) Thermo-sensitive neurons in hypothalamic tissue slices in vitro. Brain Res., 186, 203-207.

11) Inoue, S. \& Murakami, N. (1976) Unit responses in the medulla oblongata of rabbit to changes in local and cutaneous temperature. J. Physiol., 259, 339-356.

12) Mason, P., Hasan, H. \& Valis, M. (1978) Spontaneous firing of hypothalamic neurones over a narrow temperature interval. Nature, 273, 242-243.

13) Masurovsky, E.B., Benitez, H.H., \& Murray, M.R. (1971) Synaptic development in long-term organized cultures of murine hypothalamus. J. comp. Neurol., 143, 263278 .

14) Murakami, N. (1973) Effects of iontophoretic application of 5-hydroxytryptamine, noradrenaline and acetylcholine upon hypothalamic temperature-sensitive neurones in rats. Jap. J. Physiol., 23, 435-446.

15) Myhre, K. \& Hammel, H.T. (1969) Behavioral regulation of internal temperature in the lizard Tiliqua scincoides. Amer. J. Physiol., 217, 1490-1495.

16) Nakayama, T. \& Hardy, J.D. (1969) Unit responses in the rabbit's brain stem to changes in brain and cutaneous temperature. J. appl. Physiol., 27, 848-857.

17) Nakayama, T., Eisenman, J.S., \& Hardy, J.D. (1961) Single unit activity of anterior hypothalamus during local heating. Science, 134, 560-561.

18) Nakayama, T., Hammel, H.T., Hardy, J.D. \& Eisenman, J.S. (1963) Thermal stimulation of electrical activity of single units of the preoptic region. Amer. J. Physiol., 204, 1122-1126.

19) Nakayama, T., Hori, Y., Suzuki, M., Yonezawa, T. \& Yamamoto, K. (1978) Thermo-sensitive neurons in preoptic and anterior hypothalamic tissue cultures in vitro. Neurosci. Lett., 9, 23-26.

20) Nakayama, T., Ishikawa, Y. \& Tsurutani, T. (1979) Projection of scrotal thermal afferents to the preoptic and hypothalamic neurones in rats. Pflüers Arch., 380, $59-64$.

21) Reier, P.J., Cullen, M.J., Froelich, J.S. \& Rothchild, I. (1977) The ultrastructure of the developing medial preoptic nucleus in the postnatal rat. Brain Res., 122, 415436.

22) Wit, A. \& Wang, S.C. (1968) Temperature-sensitive neurons in preoptic/anterior hypothalamic region: effects of increasing ambient temperature. Amer. J. Physiol., 215, 1151-1159.

23) Yonezawa, T. (1961) Studies on myelin formation of central and peripheral nervous tissue in vitro. Acta path. jap., 11, 465-475. 